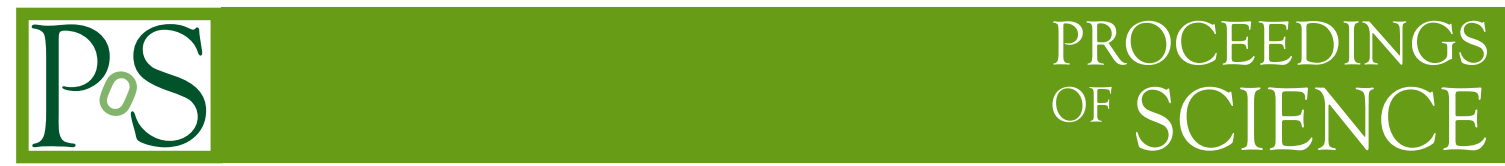

\title{
Status of "OKA" experiment
}

\author{
Victor Kurshetsov*t \\ IHEP-Protvino \\ E-mail: Victor.Kurshetsov@ihep.ru
}

Status of the "OKA"project: the experimental program with RF-separated $K^{ \pm}$beam at U-70 Protvino, Russia, is presented. The early history of the project, current stutus of the beam-line and experimental facility are shortly described. Some results (mostly technical) from recent (April 2009) run are reported. Near-term plans and possible directions of the physical program are briefly discussed.

2009 KAON International Conference

June 09 - 12, 2009

Tsukuba, Japan

${ }^{*}$ Speaker.

${ }^{\dagger}$ representing the OKA collaboration (IHEP-INR-JINR) 


\section{The history of the project}

In the late nineties of the last century IHEP, Protvino decided to start new project: RF Separated beam at IHEP U-70, based on superconducting deflectors. The only existing superconductive RF separator was built in Karlsruhe(Germany) and used at CERN SPS in 1978-1981 to provide $K^{ \pm}$ and $\bar{p} 3-37 \mathrm{GeV}$ beams for the $\Omega$-spectrometer.

In 1996 the negotiations IHEP-CERN started and in January 1998 two cryostats with niobium deflectors were transported from CERN to IHEP [1]. Meantime three major U-70 setups, working in the different areas of elementary particle physics : SPHINX (baryon spectroscopy), GAMS (meson spectroscopy) and ISTRA (kaon decays), decided to join their expertize, manpower and part of the existing equipment to create an "OKA" setup ("OKA" is a Russian abbreviation for "Experiments with Kaons"). An initial consideration of the "OKA" experimental program can be found in [2].

After the beam line design (1998) and technical project (1999) were developed, the construction phase have bean started, resulting in "warm" (unseparated) beam commissioning in December, 2004.

The cryogenic activity started with the construction of a special stand alone test facility where vacuum, helium and complex RF-tests at $4.2 \mathrm{~K}$ and $1.8 \mathrm{~K}$ can be done (1998-2004). In parallel with this work the main units of the cryogenic and vacuum plant necessary to operate the deflectors on the kaon beam were designed and manufactured at IHEP. The commissioning of the main cryogenic system started in December 2005 [3]. The overall status of the project at this stage can be found in [4].

At the same time an RF-feed and phasing system for the deflectors, based on modern microwave elements and the rubidium frequency-standard as a source of the signal was designed and implemented. The commissioning of the "cold" (separated) beam started in December 2006.

\section{The beam}

The new beam-line $(21 \mathrm{~K})$ is located in the U-70 gallery, which already houses some beams produced from the fast and slow extracted primary proton beam. It replaced the former universal hadron channel, used by the "SPHINX" experiment.

The design of optics for the separated beam was based on the existing magnetic equipment, which was developed more than 30 years ago for particle beams of U-70. It was optimized to produce a $12.5 \mathrm{GeV} / \mathrm{c}$ separated kaon beam using two deflector (Panofsky) scheme of the separation. The total length of the beam-line between the production target and the entrance of the decay region is equal to $202 \mathrm{~m}$. The r.m.s. width of momentum spread at this point is $1.5 \%$ and the size of the beam spot is $\sigma_{x} \times \sigma_{y}=34 \times 17 \mathrm{~mm}^{2}$.

Nominal parameters of the deflectors [5] and calculated parameters of the beam [6] are shown in the Tables 1 and 2 correspondingly.

There were, however, some problems with the deflectors. First of all, it turned out that one of the deflectors (RF2) was damaged at CERN during preparation for the shipment to Russia. The restoration of it's nominal working parameters has been achieved during the stand alone tests. The deflector RF1, which worked properly at this stage, showed the signs of degradation after 
it was placed at nominal position in the beam-line and connected to the main cryogenic system. The reason for such a behaviour is now fully understood and work towards the restoration of it's nominal parameters is in progress. Table 3 reflects the progress in this direction.

\begin{tabular}{|cc|}
\hline Operating frequency & $2865 \mathrm{MHz}$ \\
Wavelength, $\lambda$ & $\sim 10.5 \mathrm{~cm}$ \\
Iris opening, 2a & $40 \mathrm{~mm}$ \\
Effective deflector length & $2.74 \mathrm{~m}$ \\
Number of cells/deflector & 104 \\
Mean deflecting field & $\leq 1.2 \mathrm{MV} / \mathrm{m}$ \\
Working temperature & $1.8 \mathrm{~K}$ \\
\hline
\end{tabular}

Table 1: Nominal parameters of the deflectors

\begin{tabular}{|lc|}
\hline Target & $50 \mathrm{~cm} \mathrm{Al}$ \\
Primary proton beam energy & $65-70 \mathrm{GeV}$ \\
Primary proton beam intensity & $10^{13} \mathrm{ppp}$ \\
Secondary beam momentum & 12.5 or $18 \mathrm{GeV}$ \\
$\Delta \mathrm{p} / \mathrm{p} \%$ & \pm 4 \\
Horizontal acceptance & $\pm 10 \mathrm{mrad}$ \\
Vertical acceptance & $\pm 1.9 \mathrm{mrad}$ \\
Length of the beam line & $\sim 200 \mathrm{~m}$ \\
Distance between separators & $76.3 \mathrm{~m}$ \\
Intensity of $K^{+}$at the end & $5 \times 10^{6}$ \\
$\pi^{+}, p$ contamination & $<25 \%$ \\
Muon halo & $<100 \%$ \\
\hline
\end{tabular}

Table 2: Calculated parameters of the beam

\begin{tabular}{|l|c|c|c|c|}
\hline & Project & December 2007 & December 2008 & April 2009 \\
\hline RF1, MV/m & 1.00 & 0.32 & 0.59 & 0.55 \\
\hline RF2, MV/m & 1.00 & 0.90 & 0.92 & 0.91 \\
\hline
\end{tabular}

Table 3: Parameters of deflectors in recent runs

\section{Status of OKA setup}

Currently the installation of the OKA facility is almost completed: all the main detectors are in place, most of them are fully commissioned. The layout of the "OKA" detector is presented in Fig. 1. The right-handed $\mathrm{X}, \mathrm{Y}, \mathrm{Z}$ coordinate system of the setup has $\mathrm{Z}$-axis in the direction of the beam, vertical $\mathrm{Y}$-axis and horizontal $\mathrm{X}$-axis. The main elements of the detector are as follows:

1. Beam spectrometer [7] - magnet $\mathrm{M} 2$, seven planes of $1 \mathrm{~mm}$ pitch proportional chambers (BPC), two threshold Cherenkov counters for beam particle identification $\left(\check{\mathrm{C}}_{1}-\check{\mathrm{C}}_{2}\right)$, a set of thin scintillator counters and hodoscopes.

2. Decay volume with veto system (DV) $-11 \mathrm{~m}$ length helium filled vessel equipped with $\sim 670$ lead-scintillator sandwiches. Each sandwich has 20 layers with a structure $5 \mathrm{~mm} \mathrm{Sc}+1.5 \mathrm{~mm}$ $\mathrm{Pb}$ per layer.

3. The wide-aperture magnetic spectrometer consists of a set of proportional chambers (PC, $2 \mathrm{~mm}$ pitch), straw tubes (ST, three double planes of $1 \mathrm{~cm}$ tubes) and drift tubes (DT, two triple planes of $3 \mathrm{~cm}$ tubes). A pad hodoscope (MH), located after the last plane of the drift tubes, has $\approx 300$ readout channels and can be used as a trigger device as well as an element 


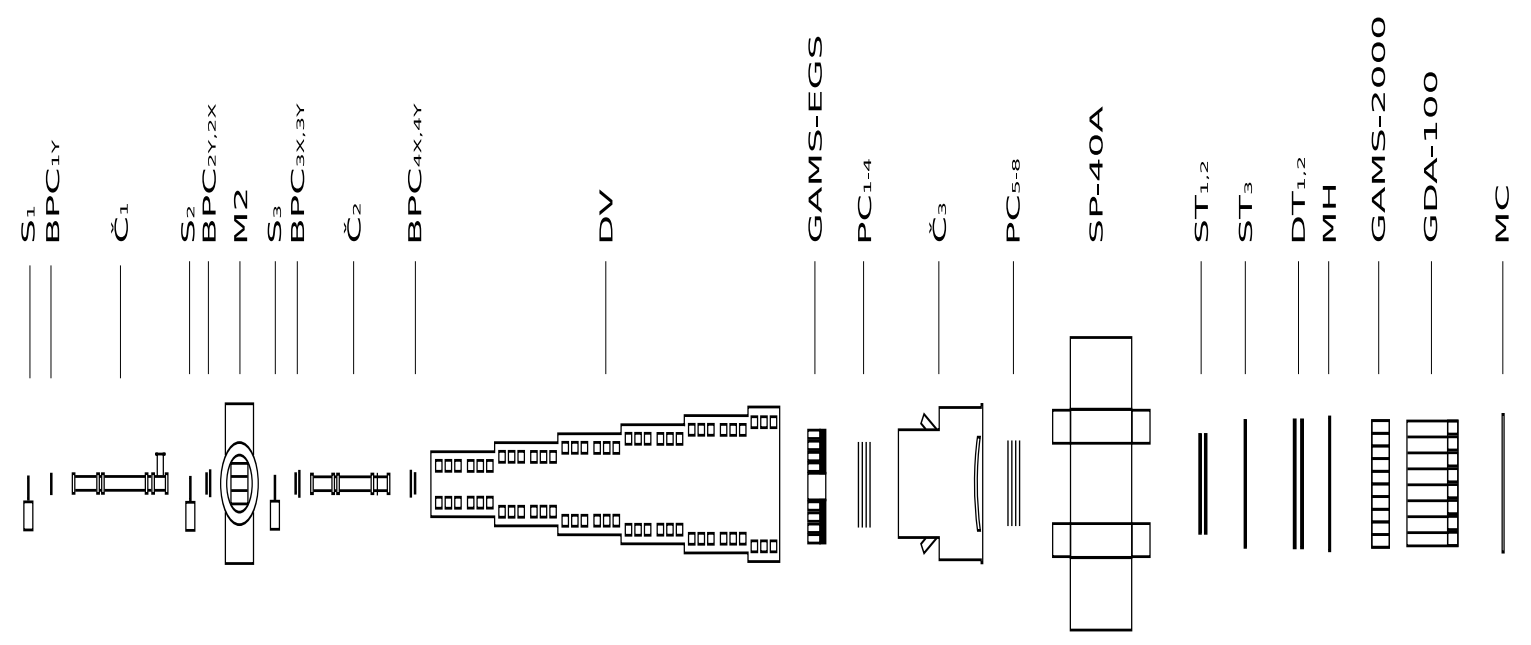

Figure 1: OKA setup

of tracking system, providing the 3D-reconstruction of charged tracks. One of the small counters of the hodoscope can be used as a beam killer (BK). A large multicell Cherenkov counter $\left(\breve{C}_{3}\right)$ is used to provide a trigger for events with electron in the final state.

4. Gamma detectors - two multichannel lead glass electromagnetic calorimeters, the main one being the well known GAMS-2000 detector, the second - significantly reworked EHS/SPHINX calorimeter, which can be used as a part of veto system as well as for the reconstruction of wide angle photons. The analog sum of amplitudes $\left(G A M S_{E}\right)$ from GAMS-2000 can be used for trigger.

5. Muon identification system currently consists of the hadron calorimeter GDA-100 and four muon trigger counters (MC).

The DAQ system is based on IHEP developed MISS standard and can provide the data transfer speed of up to $25 \mathrm{Mb} / \mathrm{sec}$. It includes about $10000 \mathrm{PC}$ channels (5 nsec shift registers), $1000 \mathrm{TDC}$ channels (0.8 nsec time resolution, based on CERN developed HPTDC chips) and about 5000 channels of ADC with $10 \mu$ sec conversion time.

It is well known that very often there is a need to perform an alternative analysis of data in order to support or reject an interesting or unusual experimental observation. That is why we decided from the very beginning to have two independent (almost) software branches, which we called Plan A and Plan B. Plan A is a development of new software based on modern technologies (C++, ROOT, GEANT4, etc.), whereas Plan B is an adoptation of well tested software based on SPHINX analysis tools (Fortran with structures, GEANT3, PAW, etc.). Below the results from Plan B implementation will be shown.

\section{OKA run April 2009}

The most recent run at OKA facility took place during first two weeks of April 2009. It was mostly technical run from U-70 point of view. In order to save the energy (and money) the 
momentum of primary proton beam was chosen to be $50 \mathrm{GeV} / \mathrm{c}$, which is not an optimal value for the separated beam. The content of the beam (25\% of kaons) was the same as in December 2008 run, as nothing was done with the deflectors in between. The stability of cryogenic system and the beam was, however, much better this time, allowing us to test and commission new equipment and collect some statistics. In fact, three days of data taking with separated $12.5 \mathrm{GeV} / \mathrm{c} K^{+}$beam and one days of calibration with $5 \mathrm{GeV} / \mathrm{c}$ electron beam resulted in total amount of data written of 830 Gb, including 300 millions physical events and 40 millions calibration events. Four simple kinds of trigger were implemented:

- Beam, $\operatorname{Tr} 1$ = prescaled coincidence of beam counters.

- Kaon decay, $\operatorname{Tr} 2=$ beam $* \overline{C_{1}} * C_{2} * \overline{B K}$

- Kaon decay with electron, $\operatorname{Tr} 3=$ beam $* \overline{C_{1}} * C_{2} * \overline{B K} * C_{3}$

- Kaon decay with photon, $\operatorname{Tr} 4=$ beam $* \overline{C_{1}} * C_{2} * \overline{B K} * G A M S_{E}$

Examples of the reconstruction of the events from two kinds of trigger are presented if Figs 2 and 3. Based on the analysis of $1 \%$ of statistics we estimate the number of reconstructable $K^{+} \rightarrow \pi^{+} \pi^{0}$ and $K^{+} \rightarrow \pi^{+} \pi^{+} \pi^{-}$decays as 2-3 $\mathrm{M}$ and 0.5-0.7 $\mathrm{M}$ events correspondingly.

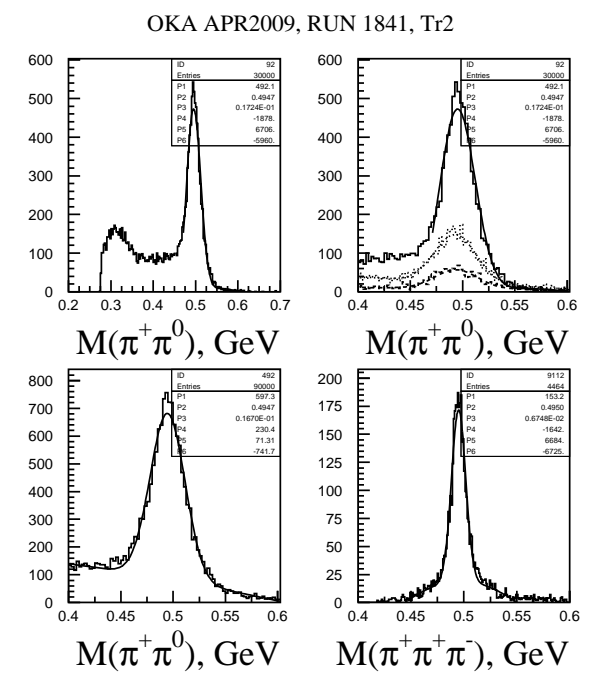

Figure 2: Reconstaruction of kaon decays for $\operatorname{Tr} 2$

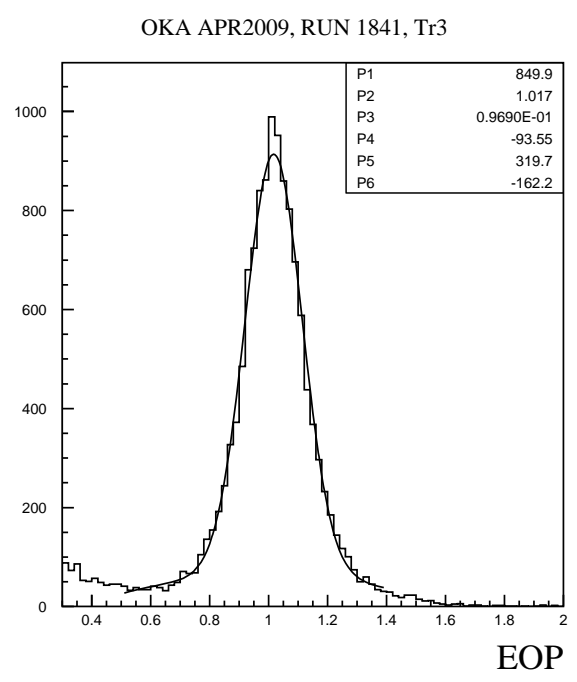

Figure 3: Electron signal (E/p) for Tr3

\section{Near-term plans and experimental program}

Next run at OKA is planned for November-December 2009. There is a plan how to improve the work of the first deflector as well as a plan how to improve beam collimation and intensity. With all that at hand we hope to have in December 1 million Kaons/spill (compare to 250k Kaons/spill in April). Meantime the data analysis should give us better understanding of the installation, better calibration and alignment constants and allow us to find the ways of various improvements. 
A few words about experimental program, which is being tuned depending on the results of the past and current experiments: ISTRA+, KLOE, E949, NA48/2, NA62, many of them were presented at this conference. It is clear however, that it will consists of two parts:

1. Kaon decays

- Continuation of the physical program of ISTRA+ setup (see the report by V. Duk at this conference [8]) with the increase in statistics by a factor of 10-100.

- ...

2. Fix-Target program

- Search for exotic mesons and baryons.

- Spectroscopy and decays of light mesons.

- Primakoff physics in kaon beam.

\section{Acknowledgments}

This work was partly supported by Russian Foundation for Basic Researches (RFBR grant 07-02-00957).

\section{References}

[1] Superconducting Separator Emerges from Sleep, CERN Courier, 38, No.3, April 1998, p.12.

[2] V. F. Obraztsov and L. G. Landsberg, Prospects for CP-violation searches in the future experiment with RF-separated K+/- beam at U-70, Nucl. Phys. Proc. Suppl. 99B, 257 (2001)

[hep-ex/0011033].

[3] A. Ageev et al., Commissioning of superconducting radiofrequency separator cryogenic system, in proceedings of 20th Russian Accelerator Conferences (RuPAC 2006), Novosibirsk, Russia, 10-14 Sept 2006.

[4] V. Obraztsov, Future kaon experiments in Russia - OKA, Prepared for International Workshop Kaon 2005, 13-17 Jun 2005, Evanston, Illinois.

[5] A. Citron, G. Dammertz, M. Grundner, L. Husson, R. Lehm and H. Lengeler, The Karlsruhe-Cern Superconducting Rf Separator, Nucl. Instrum. Meth. 164 (1979) 31.

[6] V. I. Garkusha, F. N. Novoskoltsev, V. N. Zapolsky and V. G. Zarucheisky, Calculated characteristics of the separated kaon beam for OKA experiment at the U-70 accelerator, IHEP Preprint 2003-4, Apr 2003.

[7] Akimenko S. A et al., Study of Beam Spectrometer of OKA experimental setup, IHEP Preprint 2008-28, Dec 2008 (in Russian).

[8] V. Duk, Study of K -> mu nu gamma Decay at ISTRA+ Setup, talk given at KAON09. 